\title{
Saúde bucal, fatores socioeconômicos e qualidade de vida de crianças de 12 anos de idade da cidade de Patos-PB
}

\author{
Oral health, socio-economic factors and quality of life of children 12 years old \\ in the city of Patos-PB \\ Salud bucal, factores socioeconómicos y calidad de vida de los niños de 12 años \\ de la ciudad de Patos-PB \\ Faldryene de Sousa QUEIROZ ${ }^{1}$ \\ Luciana Ellen Dantas COSTA ${ }^{1}$ \\ Tuanny Lopes Alves SILVESTRE ${ }^{2}$

\begin{abstract}
${ }^{1}$ Professora do Curso de Odontologia da Unidade Acadêmica de Ciências Biológicas, Centro de Saúde e Tecnologia Rural, UFCG - Universidade Federal de Campina Grande 58708-110 Patos - PB, Brasil UFCG - Universidade Federal de Campina Grande 58708-110 Patos - PB, Brasil
\end{abstract} \\ ${ }^{2}$ Cirurgiã-Dentista pelo Curso de Odontologia da Unidade Acadêmica de Ciências Biológicas, Centro de Saúde e Tecnologia Rural,
}

\begin{abstract}
Resumo
A cárie dentária tem sido discutida no Brasil e no mundo como um importante tema da saúde pública. O objetivo desse trabalho foi avaliar a associação entre cárie dentária, condições socioeconômicas e qualidade de vida de crianças de 12 anos de idade. O estudo transversal foi realizado com 266 crianças, de ambos os sexos, matriculadas em creches públicas e privadas da cidade de Patos/PB. Os aspectos relativos à qualidade de vida foram avaliados a partir do questionário Child Perceptions Questionnaire $\left(\mathrm{CPQ}_{11-14}\right)$. Para avaliação das condições socioeconômicas das crianças foi utilizado um formulário contendo perguntas referentes à escolaridade do chefe da família, renda mensal familiar, número de componentes no domicílio, tipo de moradia e zona do domicílio. A prevalência de cárie dentária foi avaliada por meio do índice CPO-d e seus componentes. Observou-se que 42,9\% das crianças de 12 anos tinham a doença cárie, apresentando CPO-d de 2,14, $73,5 \%$ do componente cariado. Houve associação significativa $(\mathrm{p} \leq 0,05)$ entre a cárie dentária e a qualidade de vida, bem como foi encontrada associação entre a presença de cárie e a renda, tipo de escola, escolaridade do chefe, quantidade de pessoas no domicílio e zona do domicílio. As ações de promoção e prevenção em saúde bucal devem estar cada vez mais presentes no dia a dia das crianças visando uma melhora na qualidade de vida.
\end{abstract}

Descritores: Cárie Dentária; Qualidade de Vida; Condições Sociais; Saúde Bucal.

\section{Abstract}

Tooth decay has been discussed in Brazil and in the world as an important issue of public health. The objective of this study was to evaluate an association between dental caries, socioeconomic conditions and quality of life of children from 12 years of age. The cross-sectional study was conducted with 266 children of both sexes, registered in public and private day care centers in the city of Patos / PB. The quality of life aspects were assessed using the Child Perceptions Questionnaire $\left(\mathrm{CPQ}_{11-14}\right)$ questionnaire. To make the evaluation of the socioeconomic conditions of the children was used a form and some questions related to the education of the head of the family, monthly family income, number of components not domicile, type of dwelling and area of domicile. The prevalence of dental caries was evaluated using the DMFT indices and its components (CEP / FIP-1.985.087). It was observed that $42.9 \%$ of 12 years had caries, with DMFT of 2.14 , thus, $73.5 \%$ of the carious component. There was a significant association $(\mathrm{p} \leq 0,05)$ between dental caries and quality of life in both age groups, as well as an association between the presence of caries and family income, type of school, type of housing, education of the head, number of people in the household and zone. The actions of promotion and prevention in oral health must be increasingly present in the daily life of children aiming the improvement in the quality of life.

Descriptors: Dental Caries; Quality of Life; Social Class; Oral Health.

\section{Resumen}

La caries dental ha sido discutida en Brasil y en el mundo como un importante tema de la salud pública. El objetivo de este trabajo fue evaluar la asociación entre caries, condiciones socioeconómicas y calidad de vida de niños de 12 años de edad. El estudio transversal fue realizado con 266 niños, de ambos sexos, matriculados en guarderías públicas y privadas de la ciudad de Patos / PB. Los aspectos relativos a la calidad de vida se evaluaron a partir del cuestionario Child Perceptions Questionnaire (CPQ11-14). Para la evaluación de las condiciones socioeconómicas de los niños se utilizó un formulario que contenía preguntas referentes a la escolaridad del jefe de familia, ingreso mensual familiar, número de componentes en el domicilio, tipo de vivienda y zona del domicilio. La prevalencia de la caries dental fue evaluada a través del índice CPO-d y sus componentes. Se observó que el 42,9\% de los niños de 12 años tenían la enfermedad caries, presentando CPOd de 2,14,73,5\% del componente cariado. Se observó una asociación significativa $(p \leq 0,05)$ entre la caries dental y la calidad de vida, así como se encontró asociación entre la presencia de caries y la renta, tipo de escuela, escolaridad del jefe, cantidad de personas en el domicilio y zona del distrito, casa. Las acciones de promoción y prevención en salud bucal deben estar cada vez más presentes en el día a día de los niños buscando una mejora en la calidad de vida.

Descriptores: Caries Dental; Calidad de Vida; Clase Social; Salud Bucal.

\section{INTRODUÇÃO}

A qualidade de vida relacionada à saúde está ligada ao impacto do estado de saúde sobre a capacidade do indivíduo de viver plenamente ${ }^{1}$. O crescente avanço tecnológico, que teoricamente viria a otimizar o atendimento ao paciente, culminou com a progressiva desumanização dos serviços de saúde, refletindo negativamente na qualidade de vida das pessoas. Devido a esse fato, políticas públicas de saúde foram propostas com o objetivo de proporcionar qualidade de vida para os cidadãos ${ }^{2}$.

A saúde bucal apresenta forte influência na qualidade de vida ${ }^{3}$. A Política Nacional de Promoção da Saúde propõe promover a qualidade de vida e reduzir a vulnerabilidade e riscos à saúde relacionados aos condicionantes - modos de viver, condições de trabalho, habitação, ambiente, educação, lazer, cultura, acesso a bens e serviços essenciais ${ }^{4}$. 
Em muitos países, inclusive no Brasil, ocorreu nas últimas três décadas uma diminuição significativa na prevalência e severidade da cárie dentária, devido a uma série de fatores, dentre eles, a exposição ao flúor, seja por meio de dentifrícios ou água, à ampliação da educação em saúde bucal, modificações na quantidade e na frequência do consumo de açúcar, além de um maior acesso aos serviços odontológicos ${ }^{5}$.

No entanto, a cárie dentária ainda continua a ter um caráter epidêmico e de alta prevalência ${ }^{5}$. Os resultados em nível nacional do índice de cárie em crianças de 12 anos de idade, segundo o SB Brasil 2010 foi de 2,07 e a região Nordeste apresentou aos 12 anos um CPO-D de 2,63, a meta estabelecida para essa faixa etária seria um CPO-D $<1,00$, que, no entanto, não foi alcançada ${ }^{6}$.

Os fatores sociais também têm se mostrado determinantes na condição de saúde bucal. Estudos revelam que a prevalência de cárie dentária, expressa pelo Índice CPO-D, mostra-se significativamente pior para populações de baixo nível socioeconômico $^{7-9}$. A implementação de políticas públicas visando minimizar a desigualdade social através da expansão do acesso à saúde, educação, moradia, trabalho e lazer é essencial, quando se objetiva a obtenção de uma boa qualidade de vida ${ }^{10}$.

Deste modo, considerando a relevância dos problemas bucais sobre o bem estar da criança, a presente pesquisa se propôs a verificar o impacto da condição bucal na qualidade de vida em crianças de 12 anos de idade do município de Patos-PB, bem como traçar a associação desta com o perfil socioeconômico das famílias envolvidas.

\section{MATERIAL E MÉTODO}

O presente trabalho foi aprovado no Comitê de Ética em Pesquisa com Seres Humanos das Faculdades Integradas de Patos - FIP, sob protocolo número 1.985.087. O estudo classificou-se como observacional transversal, cujo objetivo foi descrever a condição de saúde bucal de uma população, em determinado tempo e local, sendo possível também testar algumas associações. $\mathrm{O}$ universo foi constituído por crianças de 12 anos de idade, de ambos os sexos, matriculadas nas creches e escolas municipais de Patos-PB e seus respectivos pais/responsáveis.

O tamanho da amostra do estudo foi obtido por meio do processo de amostragem para população finita, segundo Cochran ${ }^{11}$, baseado nas duas faixas etárias. A margem de erro fixada e valor admitido para média e desvio-padrão foram os mesmos adotados pelo projeto Saúde bucal Brasil 2003 (Projeto SB Brasil) para a região Nordeste, para crianças de 12 anos de idade, em população maior de 100 mil habitantes. Considerando um percentual de perda de até $20 \%$ chegou-se a uma amostra final de 266 crianças avaliadas.

Utilizou-se como critério de inclusão para o estudo, as crianças de 12 anos de idade regularmente matriculadas em creches e escolas da rede pública e privada de Patos/PB, cujos pais assinaram o termo de consentimento livre e esclarecido. Como critério de exclusão considerou-se que crianças que apresentassem deficiências neuropsicomotoras, ou seja, desvios de normalidade de ordem mental, física, sensorial e comportamental e as crianças que não permitissem a realização do exame clínico ou que fossem vetados pelos pais/responsáveis.

Previamente à coleta do índice CPO-D e aplicação dos questionários, realizou-se uma calibração (Kappa=0,81-0,91 inter-examinadores e de 0,83-0,90 intra-examinadores), sendo utilizada a metodologia proposta por Antunes e Peres ${ }^{12}$ (2006). A verificação da concordância intra-examinador foi realizada antes e também durante a coleta dos dados, no sentido de aferir se a consistência obtida no treinamento foi mantida em campo.

Para avaliação do impacto da saúde bucal na qualidade de vida das crianças de 12 anos de idade foi utilizada a versão brasileira do questionário Child Perception Questionnaire- $\left(\mathrm{CPQ}_{11-14}\right)$ Versão reduzida (ISF:16) ${ }^{13}$, o qual contém perguntas sobre a classificação global de saúde bucal da criança e do grau em que a condição bucal afeta seu bem estar geral e perguntas sobre a frequência de eventos nos últimos três meses relacionadas com a saúde bucal, organizado em quatro domínios: sintomas de saúde oral, limitações funcionais, bem estar emocional e bem estar social.

$\mathrm{O}$ instrumento $\mathrm{CPQ}_{11-14}$ foi respondido pela própria criança (autorrelato), em sala reservada, sob supervisão do pesquisador, após explicações previamente definidas a todos os participantes.

Para avaliação das condições de saúde bucal dos escolares foi utilizado o índice CPO-D e necessidade de tratamento para cárie dentária. Os exames clínicos foram realizados por um examinador e anotador previamente calibrados. As crianças foram examinadas nas dependências das creches e escolas, em local sob iluminação natural, ventilado e próximo de uma fonte de água, estando examinador e criança sentados em cadeiras.

Os índices de cárie dentária e necessidade de tratamento foram utilizados segundo os códigos e critérios recomendados pela Organização Mundial da Saúde (OMS), na publicação Oral Healthsurveys: Basicmethods, quarta edição, no ano de 1997, adaptados pelo projeto SB Brasil 2003. Os dados obtidos foram anotados em fichas próprias para facilitar a tabulação dos dados seguindo metodologia proposta pelo SB Brasil $2010^{6}$.

Para classificação socioeconômica do núcleo 
familiar foram avaliados o nível de escolaridade do chefe da família, renda mensal familiar ${ }^{14}$, número de membros da família que participam e /ou dependem da referida situação econômica ${ }^{15}$, tipo de escola $^{16}$ condição de moradia ${ }^{14}$ e localização do domicílio.

Os alunos detectados com a presença de dentes cariados ou com necessidade de tratamento tiveram seus pais e/ou responsáveis informados e receberam encaminhamento para a Unidade Básica de Saúde correspondente a área da abrangência do domicílio ou para a clínica escola de Odontologia da Universidade Federal de Campina Grande (UFCG).

Os dados foram estratificados de acordo com a idade e gênero e analisados e processados por meio de planilhas Excel (®Microsoft 2013). A análise estatística foi realizada de modo descritivo por meio de frequências relativas e absolutas para as variáveis categóricas e os testes de Qui-quadrado e Correlação de Spearman foram utilizados para testar as associações, sendo adotado um nível de significância de $5 \%$. Os testes foram calculados por meio do programa SPSS ${ }^{\circledR}$ (StatisticalPackage for the Social Sciences) versão 21.0 para Microsoft Windows.

\section{RESULTADOS}

\section{- Caracterização da amostra}

Participaram da pesquisa um total de 266 crianças de 12 anos de idade e seus respectivos pais/responsáveis, correspondendo a $95 \%$ do total selecionado por meio do cálculo amostral, 01 criança não participou do estudo por resposta incompleta do questionário, 11 foram excluídas por apresentarem os termos de consentimento livre e esclarecido sem a autorização do responsável e 02 pela ausência da criança na escola no dia da realização do exame clínico.

Observou-se que das crianças participantes, $62,8 \%$ eram do sexo feminino $(n=167)$ e $37,2 \%$ era do masculino $(\mathrm{n}=99)$, sendo $51,1 \% \quad(\mathrm{n}=136)$ provenientes de escolas públicas e 48,9\% $(\mathrm{n}=130)$ de escolas particulares.

Em relação ao perfil socioeconômico das famílias dos escolares, constatou-se que 33,1\% (n= 88) dos familiares tinham uma renda familiar de menos de 1 salário mínimo, 37,2\% (n=99) entre 1 e 2 salários mínimos e 29,7\% (n=79) recebiam mais de 3 salários mínimos.

Percebeu-se que $89,1 \%(\mathrm{n}=237)$ dos chefes das famílias eram alfabetizados, destes, 19,9\% $(\mathrm{n}=53)$ tinham ensino fundamental incompleto, $17,7 \% \quad(n=47)$ haviam completado o ensino fundamental, 24,8\% $(n=66)$ cursaram até o ensino médio e $26,7 \%$ ( $n=71)$ o ensino superior. Quanto ao número de moradores no domicílio, observou-se percentuais que variavam de $2(3,8 \%)$ a $11(0,4 \%)$ pessoas. A maioria das famílias participantes do estudo eram compostas por quatro pessoas 33,8\% $(\mathrm{n}=90), 91,7 \% \quad(\mathrm{n}=244)$ viviam na zona urbana, $53,4 \%(n=142)$ moravam em casa própria e $84,6 \%$ $(\mathrm{n}=225)$ relataram ter acomodação suficiente para todos os integrantes da família.

- Avaliação do perfil epidemiológico da cárie dentária em escolares de 12 anos de idade

Ao analisar o perfil epidemiológico da cárie dentária podemos observar um CPO-d médio de 2,14 dentes acometidos e valores mínimo e máximo de 1 e 15, na devida ordem. Foi observado que $42,9 \%(n=114)$ das crianças apresentavam ao menos a presença de uma lesão de cárie, totalizando 570 dentes afetados, destes, $73,5 \%(\mathrm{n}=419)$ era do componente cariado, $19,8 \%(\mathrm{n}=113)$ restaurados e $6,7 \%(\mathrm{n}=38)$ extração indicada ou perdido devido a cárie.

Em relação a distribuição do número de dentes acometidos pela doença cárie por criança, percebeu-se que $33,4 \%$ dos escolares apresentavam de 1 a 5 dentes com experiência de cárie e 9,5\% mais de 6 dentes acometidos pela cárie.

- Avaliação da influência da condição de saúde bucal na qualidade de vida dos escolares

Observou-se através dos dados obtidos por meio da aplicação do $\mathrm{CPQ}_{11-14}$ que a amplitude de respostas às questões variaram de 0 a 43 (de um total de 64 pontos) com média de 12,8 ( $\mathrm{DP}=8,6)$. A prevalência de crianças com qualidade de vida prejudicada foi de $98,1 \%(\mathrm{n}=261)$. Observou-se associação entre a presença de cárie dentária e a influência da saúde bucal na qualidade de vida (Tabela 1).

Tabela 1. Presença de Cárie dentária em função da qualidade de vida relacionada à saúde bucal de crianças de 12 anos de idade, Patos, 2018

\begin{tabular}{|c|c|c|c|c|}
\hline \multirow[b]{2}{*}{ Variável } & \multicolumn{3}{|c|}{ CÁRIE DENTÁRIA } & \multirow[b]{2}{*}{$\mathbf{P}^{(1)}$} \\
\hline & Ausente & Presente & Total & \\
\hline & n (\%) & $\mathrm{n}(\%)$ & $\mathrm{n}(\%)$ & \\
\hline \multicolumn{5}{|c|}{ Child Perception Questionnaire- $\left(\mathrm{CPQ}_{11-14}\right)$} \\
\hline Prejudicada & $147(96,7)$ & $114(100)$ & $261(100,0)$ & 0,050 \\
\hline $\begin{array}{c}\text { Não } \\
\text { prejudicada }\end{array}$ & $5(3,3)$ & $0(0)$ & $5(100,0)$ & \\
\hline
\end{tabular}

Quando as crianças foram questionadas sobre como consideravam a saúde dos dentes, lábios, boca e maxilares, $37,6 \% \quad(n=100)$ consideravam boa e 28,9\% $(n=77)$ regular. Em relação a dor, 40,2\% $(\mathrm{n}=107)$ relataram não ter sentido dor nos últimos 3 meses, e $27,1 \%(n=72)$ uma ou duas vezes.

A Tabela 2 mostra as médias por quesito e a distribuição por domínios do $\mathrm{CPQ}_{11-14}$.

Houve relação estatisticamente significante entre a presença de cárie dentária e a qualidade de vida $(\mathrm{p}=0,050)$ (Tabela 1), e alguns quesitos isolados 
mostraram também essa associação: "a criança teve dor nos últimos 3 meses? " $(\mathrm{p}=0,000)$, assim como em "alimentos presos entre os dentes após as refeições" $(p=0,016)$, "se sentiu constrangido ou com vergonha por causa dos dentes, lábios, boca ou maxilares" $(\mathrm{p}=0,036)$, "dificuldade para morder ou mastigar alimentos duros como espiga de milho, maçã" ( $p=0,014)$, "outras crianças aborreceram ou chamaram por apelidos" $(\mathrm{p}=0,001)$.

Tabela 2. Média aritmética dos escores por quesito e domínios do $\mathrm{CPQ}_{11-14}$, Patos, 2018

\begin{tabular}{lcl}
\hline $\begin{array}{l}\text { Quesitos do } C P Q_{11-14} \text { por } \\
\text { domínio }\end{array}$ & $\begin{array}{c}\text { Média dos } \\
\text { escores por } \\
\text { quesito* }\end{array}$ & $\begin{array}{l}\text { Média por } \\
\text { domínio }\end{array}$ \\
\hline
\end{tabular}

\begin{tabular}{|c|c|c|}
\hline \multicolumn{3}{|c|}{ Sub-escala CRIANÇA } \\
\hline \multicolumn{3}{|c|}{ Domínio dos Sintomas Orais } \\
\hline $\begin{array}{l}\text { 1.Dor nos dentes, lábios, } \\
\text { maxilares ou boca? }\end{array}$ & 1,01 & \\
\hline 2.Feridas na boca? & 0,53 & \\
\hline 3.Mau hálito? & 1,04 & 1,0 \\
\hline $\begin{array}{l}\text { 4. Restos de alimentos } \\
\text { presos dentro ou entre os } \\
\text { dentes? }\end{array}$ & 1,42 & \\
\hline \multicolumn{3}{|c|}{ Domínio das limitações funcionais } \\
\hline $\begin{array}{l}\text { 5.Demorou mais que os } \\
\text { outros para terminar a } \\
\text { refeição? }\end{array}$ & 1,01 & \\
\hline $\begin{array}{l}\text { 6.Dificuldade para morder } \\
\text { ou mastigar alimentos } \\
\text { duros como maçãs, espiga } \\
\text { de milho ou carne? }\end{array}$ & 0,59 & \\
\hline $\begin{array}{l}\text { 7.Dificuldade para dizer } \\
\text { alguma palavra? }\end{array}$ & 0,67 & 0,84 \\
\hline $\begin{array}{l}\text { 8. Dificuldade para beber ou } \\
\text { comer alimentos quentes } \\
\text { ou frios? }\end{array}$ & 1,11 & \\
\hline
\end{tabular}

\begin{tabular}{lcc}
\hline \multicolumn{3}{c}{ Domínio do Bem Estar Emocional } \\
\hline $\begin{array}{l}\text { 9.Ficou irritado(a) ou } \\
\text { frustrado(a)? }\end{array}$ & 0,60 \\
$\begin{array}{l}\text { 10.Ficou tímido, } \\
\text { constrangido ou com } \\
\text { vergonha? }\end{array}$ & 0,81 & \\
$\begin{array}{l}11 . \text { Ficou chateado? } \\
\text { 12.Ficou preocupado com }\end{array}$ & 0,73 & \\
$\begin{array}{l}\text { o que outras pensam sobre } \\
\text { seus dentes, lábios, boca } \\
\text { ou maxilares? }\end{array}$ & 0,87 & \\
\hline
\end{tabular}

\begin{tabular}{ll}
\hline \multicolumn{3}{c}{ Domínio do Bem Estar Social } \\
\hline $\begin{array}{l}\text { 13.Evitou sorrir ou dar } \\
\text { risadas quando está com } \\
\text { outras crianças? }\end{array}$ \\
$\begin{array}{l}\text { 14.Discutiu com outras } \\
\text { crianças ou pessoas da } \\
\text { família? }\end{array}$ \\
$\begin{array}{l}\text { 15.Outras crianças } \\
\text { aborreceram ou chamaram }\end{array}$ \\
por apelidos? \\
$\begin{array}{l}\text { 16.Outras crianças fizeram } \\
\text { perguntas sobre seus } \\
\text { dentes, lábios, maxilares e } \\
\text { boca? }\end{array}$ \\
*0-Nunca; 4-Todos os dias ou quase todos os dias
\end{tabular}

A avaliação da qualidade de vida das crianças não apresentou relação estatisticamente significante com nenhuma variável socioeconômica: sexo $(\mathrm{p}=0,421)$, renda mensal familiar $(\mathrm{p}=0,325)$, número de residentes por domicílio ( $\mathrm{p}=0,467)$, escolaridade do responsável $(\mathrm{p}=0,430)$, situação da habitação ( $\mathrm{p}=0,791)$, localização da residência $(\mathrm{p}=0,336)$, ou tipo de escola da criança $(\mathrm{p}=0,430)$.

- Cárie dentária e fatores socioeconômicos em crianças de 12 anos de idade

Na Tabela 3, pode-se observar por meio de análise bivariada, a prevalência de cárie dentária em escolares de 12 anos em relação às variáveis de ordem socioeconômicas, pelo teste Qui-Quadrado $(\mathrm{p} \leq 0,05)$.

Tabela 3. Associação entre a presença de cárie dentária e os fatores socioeconômicos em crianças de 12 anos de idade, Patos, 2018

\begin{tabular}{|c|c|c|c|c|}
\hline \multicolumn{5}{|c|}{ CÁRIE DENTÁRIA } \\
\hline \multirow[t]{2}{*}{ Variável } & Ausente & Presente & Total & $\begin{array}{l}\text { Valor } \\
\text { de } p\end{array}$ \\
\hline & $\mathrm{n}(\%)$ & $\mathrm{n}(\%)$ & $\mathrm{n}(\%)$ & \\
\hline \multicolumn{5}{|c|}{ VARIÁVEIS CRIANÇAS } \\
\hline Masculino & $57(57,6)$ & $42(42,4)$ & $99(100,0)$ & 0,913 \\
\hline Feminino & $95(56,9)$ & $72(43,1)$ & $167(100,0)$ & \\
\hline \multicolumn{5}{|c|}{ VARIÁVEIS SOCIOECONÔMICAS } \\
\hline \multicolumn{5}{|c|}{$\begin{array}{l}\text { Escolaridade } \\
\text { do Chefe da } \\
\text { Família }\end{array}$} \\
\hline Analfabeto & $8(27,6)$ & $21(72,4)$ & $29(100,0)$ & 0,003 \\
\hline $\begin{array}{l}\text { Ensino } \\
\text { fundamental } \\
\text { incompleto }\end{array}$ & $26(49,1)$ & $27(50,9)$ & $53(100,0)$ & \\
\hline $\begin{array}{l}\text { Ensino } \\
\text { fundamental } \\
\text { completo }\end{array}$ & $26(55,3)$ & $21(44,7)$ & $47(100,0)$ & \\
\hline Ensino médio & $39(59,1)$ & $27(40,9)$ & $66(100,0)$ & \\
\hline $\begin{array}{l}\text { Ensino } \\
\text { superior }\end{array}$ & $53(74,6)$ & $18(25,4)$ & $71(100,0)$ & \\
\hline \multicolumn{5}{|l|}{$\begin{array}{l}\text { Renda } \\
\text { Mensal } \\
\text { Familiar }\end{array}$} \\
\hline$<1 \mathrm{SM}^{*}$ & $37(42,0)$ & $51(58,0)$ & $88(100,0)$ & 0,001 \\
\hline 1- $2 \mathrm{SM}^{*}$ & $57(57,6)$ & $42(42,4)$ & $99(100,0)$ & \\
\hline$\geq 3 \mathrm{SM}^{*}$ & $58(73,4)$ & $21(26,6)$ & $79(100,0)$ & \\
\hline \multicolumn{5}{|l|}{$\begin{array}{l}\text { Pessoas no } \\
\text { Domicílio }\end{array}$} \\
\hline$<6$ pessoas & $142(58,9)$ & $99(41,1)$ & $241(100,0)$ & 0,002 \\
\hline$\geq 6$ pessoas & $10(40,0)$ & $15(60,0)$ & $25(100,0)$ & \\
\hline \multicolumn{5}{|l|}{$\begin{array}{l}\text { Tipo de } \\
\text { Escola }\end{array}$} \\
\hline Pública & $57(41,9)$ & $79(58,1)$ & $136(100,0)$ & 0,001 \\
\hline Privada & $95(73,1)$ & $35(26,9)$ & $130(100,0)$ & \\
\hline \multicolumn{5}{|l|}{$\begin{array}{l}\text { Zona } \\
\text { Domicíliar }\end{array}$} \\
\hline Rural & $7(31,8)$ & $15(13,2)$ & $22(100,0)$ & 0,012 \\
\hline Urbana & $145(59,4)$ & $99(40,6)$ & $244(100,0)$ & \\
\hline \multicolumn{5}{|l|}{$\begin{array}{l}\text { Tipo de } \\
\text { Moradia }\end{array}$} \\
\hline Própria & $85(59,9)$ & $57(40,1)$ & $142(100,0)$ & \\
\hline Financiada & $17(70,8)$ & $8(29,2)$ & $25(100,0)$ & \\
\hline Alugada & $45(54,4)$ & $36(45,6)$ & $81(100,0)$ & 0,062 \\
\hline Cedida & $5(27,8)$ & $13(72,2)$ & $18(100,0)$ & \\
\hline
\end{tabular}




\section{DISCUSSÃO}

\section{- Prevalência de Cárie Dentária}

A cárie dentária ainda é um problema de saúde pública em todo o mundo, e estudos mostram que a causa também está associada a fatores socioeconômicos, devido à dificuldade ou impossibilidade de acesso aos serviços públicos ${ }^{17}$. Embora Bastos et al. ${ }^{18}$ relatem o seu declínio nas últimas décadas, ele ainda não é tão homogêneo como podem supor os levantamentos epidemiológicos, por ser, simplesmente, uma comparação de médias. O que de fato acontece é a polarização da doença, com concentração e aumento da necessidade de tratamento em uma pequena parcela da população ${ }^{14}$.

Observou-se número elevado de crianças de 12 anos de idade com cárie dentária, corroborando estudos que citam altos valores de prevalência ${ }^{19,20}$. Divergindo da presente pesquisa, o estudo de Alves et al. ${ }^{21}$ mostra uma baixa prevalência da doença cárie.

Em relação aos dados de cárie dentária nas crianças de 12 anos, a porcentagem de crianças livres de cárie em Patos-PB é menor do que o valor nacional $(43,5 \%)$ e maior que a Região Nordeste $(37,7 \%)^{6}$.

Ao avaliar os resultados do SB Brasil $2010^{6}$ foi constatado que uma criança brasileira aos 12 anos apresenta um CPO-d médio de 2,07, dados aquém aos encontrados nesta pesquisa. No entanto, o valor do CPO-D nas cidades do interior do Nordeste foi de 3,84 , resultado além do encontrado no presente estudo. Estudos semelhantes a este também encontrou CPO-d abaixo da meta preconizada pela $\mathrm{OMS}^{22,23}$.

\section{- Carie Dentária e Fatores Socioeconômicos:}

Neste estudo, optou-se por avaliar, associado à prevalência da cárie dentária, os dados socioeconômicos das famílias envolvidas. Os fatores socioeconômicos apresentam forte influência sobre a condição de saúde bucal ${ }^{16,24,25}$, afirmação também comprovada neste trabalho. Em estudos semelhantes, Meneghim et al. ${ }^{26}$ não identificaram associação entre presença de cárie e nenhum fator socioeconômicos, em contrapartida aos resultados observados por Mazzillo et al. ${ }^{27}$ que identificaram associação entre a presença de cárie e o tipo de escola da criança. Corrêa-Faria et $\mathrm{al}^{28}$ encontraram associação significativa entre a presença de cárie e a renda familiar; Oliveira et al. ${ }^{24}$ associaram a presença de cárie ao número de residentes por domicílio, escolaridade do chefe e tipo de escola da criança e Piovesan et al. $^{7}$ afirmaram que a renda e a escolaridade do chefe são os fatores socioeconômicos de maior associação com a presença da cárie dentária.

Os escolares de 12 anos de idade que estudavam em escolas públicas apresentaram maior prevalência de cárie dentária, resultados também encontrado no estudo de Freire et al. ${ }^{29}$ enquanto que as crianças que estudavam em escolas particulares, moravam em casas próprias e cujas famílias possuíam renda mensal acima de 3 salários mínimos, apresentaram os menores índices de aparecimento da doença. Estudos prévios conduzidos no Brasil encontraram associação entre experiência de cárie, renda familiar, escolaridade da mãe e impactos na QVRSB de crianças tanto de escolas públicas ${ }^{16,17}$ quanto privadas ${ }^{17}$.

A presença de cárie em relação ao sexo não mostrou relevância significativa neste trabalho, resultado também encontrado em Castillo et al. ${ }^{30}$.

\section{- Cárie Dentária e Qualidade de Vida}

Por meio da aplicação do $\mathrm{CPQ}_{11-14}$, observouse uma associação entre cárie dentária e qualidade de vida, resultado também encontrado em outros estudos $^{2,25,31}$, divergindo do estudo de Menezes et al. ${ }^{32}$ que não encontrou associação estatisticamente significante. Alguns quesitos isolados também mostraram associação com a presença de cárie dentária, como "restos de alimentos presos entre os dentes", esta foi a questão que obteve maior média de resposta incluída no domínio dos sintomas orais e este achado pode ser comprovado também em Alves e Gonçalves ${ }^{33}$.

Os principais impactos da condição oral na qualidade de vida dos participantes neste estudo relacionam-se com "Sintomas Orais" e as "Limitações Funcionais", esses dois domínios também foram os mais prevalentes em outro estudo ${ }^{32}$, enquanto que o domínio do bem-estar foi o de menor impacto, divergindo de outros estudos ${ }^{25,31}$.

\section{- Qualidade de vida e fatores socioeconômicos}

$\mathrm{O}$ presente estudo não identificou associação estatisticamente significante entre os fatores socioeconômicos e a qualidade de vida, diferentemente de outros estudos ${ }^{34,35}$. No entanto, pôde-se constatar que tanto as crianças, como seus familiares, apresentam a qualidade de vida comprometida em função das alterações bucais. Sabendo-se, portanto, que a condição de saúde bucal sofre bastante influência dos fatores socioeconômicos, como já abordado anteriormente, não se pode dissociar a avaliação da qualidade de vida das condições socioeconômicas.

\section{CONCLUSÃO}

Os resultados do presente estudo constataram uma alta prevalência de cárie dentária na população estudada, com percentual elevado do componente não tratado da doença. Observou-se associação entre a ocorrência da cárie e os fatores socioeconômicos, além da forte influência da condição de saúde bucal na qualidade de vida da criança. Estes dados chamam a atenção para a necessidade de políticas públicas voltadas para essa população com ênfase em ações 
educativas, preventivas, assistenciais e de promoção a saúde, direcionadas à melhoria das condições de saúde bucal e, consequentemente, da qualidade de vida da criança.

\section{REFERÊNCIAS}

1. Gottardo AC, Webber LP, Rossa J, Arossi GA. Perda dental anterior influencia a qualidade de vida relacionada à saúde oral. Rev Inic Científica ULBRA. 2015; 13:60-70.

2. Fleck MPA, Leal OF, Louzada S, Xavier M, Chachamovic E, Vieira $G$ et al. Desenvolvimento da versão em português do instrumento de avaliação de qualidade de vida da OMS (WHOQOL-100). Rev Bras Psiquiatr. 1999; 21(1):19-28.

3. Paula JS, Cruz JN, Ramires TG, Ortega EMM, Mialhe FL. Longitudinal impact of clinical and socioenvironmental variables on oral healthrelated quality of life in adolescents. Braz Oral Res. 2017; 31:e70.

4. Brasil. Política Nacional de Promoção da Saúde. Brasília: Ministério da Saúde 2006.

5. Rebelo STCP, Santanna GR. Prevalência de cárie dental em escolares de 12 anos na rede municipal de ensino de Parnaíba Piauí. Rev Interd Ciên Saúde. 2015; 2(3):11-18.

6. Brasil. Ministério da Saúde. Secretaria de Atenção à Saúde. Secretaria de Vigilância em Saúde. SB Brasil 2010: Pesquisa Nacional de Saúde Bucal: resultados principais / Ministério da Saúde.2011; Secretaria de Atenção à Saúde. Secretaria de Vigilância em Saúde. - Brasília : Ministério da Saúde.

7. Piovesan C, Tomazoni F, Del Fabro J, Buzzati BC, Mendes FM, Antunes JL et al. Inequality in dental caries distribution at noncavitated and cavitated thresholds in preschool children. J Public Health Dent.2014; 74(2):120-26.

8. Figueiredo MC, Peixoto LT, Silva KVCL, Jardim LE. Saúde Bucal de Pessoas em Situação de Pobreza Extrema Residentes em um Município no Sul do Brasil. UNOPAR Cient Ciênc biol saúde. 2014; 16(1):45-50.

9. Brizon VC, Melo RR, Zarzar PM, Gomes VE, Oliveira ACB. Indicadores socioeconômicos associados à cárie dentária: uma revisão crítica. Unimontes Científica. 2015; 16(1):79-91.

10. Scarpelli AC, Paiva SM, Viegas CM, Carvalho AC, Ferreira FM, Pordeus IA. Oral health-related quality of life among Brazilian preschool children. Community Dent Oral Epidemiol. 2013; 41(4):336-44.

11.Cochran WG. Sampling techniques. 3th.ed. Edition. New : Wiley; 1977.

12. Antunes JLF, Peres MA, Frazão P. Cárie dentária in: Antunes JLF, Peres MA. Epidemiologia da saúde bucal. Rio de Janeiro: Guanabara Koogan; 2006. p.49-67.

13. Bendo CB, Paiva SM, Torres CS, Oliveira AC, Goursand D, Pordeus IA. Association between treated/untreated traumatic dental injuries and impacto na quality of life of Brazilian schoolchildren. Health Qual Life Outcomes. 2010; 8:114.

14. Oliveira LB, Sheiham A, Bönecker M. Exploring the association of dental caries with social factors and nutritional status in Brazilian preschool children. Eur J Oral Sci. 2008;116(1):37-43.

15. Melo MMDC, Souza WV, Lima MLC, Braga C. Fatores associados à cárie dentária em préescolares do Recife, Pernambuco, Brasil. Cad Saude Publica. 2011; 27(3):471-85.

16.Piovesan C, Antunes JL, Guedes RS, Ardenghi TM. Impact of socioeconomic and clinical factors on child oral health-related quality of life (COHRQoL). Qual Life Res. 2010;19(9):1359-66.

17.Schuch HS, Costa FS, Torriani DD, Demarco FF, Goettems ML. Oral health-related quality of life of schoolchildren: impact of clinical and psychosocial variables. Int J Paediatr Denti.2015; 25(5):358-65.

18. Bastos JLD, Nomura LH, Peres MA. Tendência de cárie dentária em escolares de 12 e 13 anos de idade de uma mesma escola no período de 1971 a 2002, em Florianópolis, Santa Catarina, Brasil. Cad Saúde Pública. 2004; 20(1):117-22.

19.Azevedo AC, Valença AMG, Lima Neto EA. Perfil epidemiológico da cárie dentária em escolares de 5 e 12 anos residentes no Município de Bayeux, Paraíba. Arq Odontol. 2012; 48(02): 68-75.

20.Dourado MR, Rebelo JHA, Rocha AL, SantaRosa TTA. Prevalência de cárie em escolares da zona rural de Indaiabira, Minas Gerais, Brasil. Rev APS. 2017; 20(1):89-97.

21.Alves NS, Gavina VP, Robles FRP, Silveira FM, Assaf AV. Determinantes Sociais e sua Relação com a Experiência de Cárie em Adolescentes: um Estudo Transversal. UNOPAR Cient Ciênc biol saúde. 2015; 17(2):93-9.

22. Oliveira LB, Moreira RS, Reis SCGB, Freire MCM. Cárie dentária em escolares de 12 anos: análise multinível dos fatores individuais e do ambiente escolar em Goiânia. Rev bras epidemiol. 2015; 18(3):642-54.

23. Muller IB, Castilhos ED, Camargo MBJ, Goncalves H. Experiência de cárie e utilização do serviço público odontológico por escolares: estudo descritivo em Arroio do Padre, Rio Grande do Sul, 2013. Epidemiol Serv Saúde. 2015; 24(4):759-70.

24. Oliveira LJ, Correa MB, Nascimento GG, Goettems ML, Tarquinio SB, Torriani DD et al. 
Iniquidade em saúde bucal: escolares beneficiaries do Bolsa Família são mais vulneráveis? 2013; 47(6):1039-47.

25.Eskenazi EMS, Sousa KG, Agostini LTP, Barbosa TS, Castelo PM. Avaliação da experiência de cárie e qualidade de vida relacionada à saúde bucal de escolares. Rev Bras Promoç Saúde. 2015; 28(2):198-205.

26. Meneghim, MC, Kozlowski FC, Pereira AC, Ambrosano GMB, Meneghim ZMAP. Classificação socioeconômica e sua discussão Ciênc saúde coletiva. 2007; 12(2):523-29.

27.Mazzillo MM, Almeida ER, Duarte DA, Sant'Anna GR. Cárie dentária relacionada às condições sócio-econômicas e qualidade de vida na infância. Arq Med Hosp Fac Cienc Med Santa Casa São Paulo. 2008; 53(3):107-12.

28.Corrêa-Faria P, Martins-Júnior PA, VieiraAndrade RG, Marques LS, Ramos-Jorge ML. Factors associated with the development of early childhood caries among Brazilian preschoolers. Braz Oral Res. 2013; 27(4):356-62.

29. Freire MCM, Reis SCGB, Gonçalves MM, Balbo PL, Leles CR. Condição de saúde bucal em escolares de 12 anos de escolas públicas e privadas de Goiânia, Brasil. Rev Panam Salud Publica. 2010; 28(2):86-91.

30. Castillo LO, Moro RC, Zanatta FB, Flores DM. Prevalência de cárie dentária nos alunos da Escola Municipal Adelmo Simas Genro, Santa Maria, RS: uma análise descritiva parcial. Dis Scientia. 2016; 7(1):121-25.

31.Fernandes MLMF, Moura FMP, Gamaliel KS, Correa-Farias P. Cárie dentária e necessidade de tratamento ortodôntico: impacto na qualidade de vida de escolares. Pesq Bras Odontoped Clin Integr. 2013; 13(1):37-43.

32. Menezes KE, Pereira CAS, Pedro ACB, Dias AGA. Avaliação do impacto da doença cárie na qualidade de vida de crianças com faixa etária de 6 a 12 anos, atendidas na clínica odontológica da Faculdade São Lucas. Rev Odontol Univ Cid São Paulo. 2009; 21(1):24-30.

33. Alves D, Gonçalves A. Impacto da saúde oral na qualidade de vida de jovens entre os 11 e os 14 anos. Revista da Faculdade de Ciencias da Saúde. Porto: Edições Fernando Pessoa; 2009.

34. Abanto J, Carvalho TS, Bönecker M, Ortega AO, Ciamponi AL, Raggio DP. Parental reports of the oral health-related quality of life of children with cerebral palsy. BMC Oral Health. 2012; 12:15.

35. Kumar S, Kroon J, Lalloo R. A systematic review on the impact of parental socio-economic status and home environment characteristics on children's oral health related quality of life. Health Qual Life Outcomes. 2014; 12:41.

\section{CONFLITO DE INTERESSES}

Os autores declaram não haver conflitos de interesse.

\section{AUTOR PARA CORRESPONDENCIA}

\section{Faldryene de Sousa Queiroz}

falqueiroz@hotmail.com

Submetido em 29/05/2018

Aceito em 27/06/2018 